\title{
Control-Matrix Approach to Stellarator Design and Control
}

\author{
H.E. Mynick, N. Pomphrey \\ Plasma Physics Laboratory, Princeton University \\ P.O. Box 451 \\ Princeton, New Jersey 08543-0451, U.S.A.
}

\begin{abstract}
The full space $\mathbf{Z} \equiv\left\{Z_{j=1, \ldots N_{z}}\right\}$ of independent variables defining a stellarator configuration is large. To find attractive design points in this space, or to understand operational flexibility about a given design point, one needs insight into the topography in $\mathbf{Z}$-space of the physics figures of merit $P_{i}$ which characterize the machine performance, and means of determining those directions in $\mathrm{Z}$-space which give one independent control over the $P_{i}$, as well as those which affect none of them, and so are available for design flexibility. The control matrix (CM) approach described here provides a mathematical means of obtaining these. In this work, we describe the CM approach and use it in studying some candidate Quasi-Axisymmetric (QA) stellarator configurations the NCSX design group has been considering. In the process of the analysis, a first exploration of the topography of the configuration space in the vicinity of these candidate systems has been performed, whose character is discussed.
\end{abstract}

PACS \#s: 52.55.Hc, 52.25.Fi, 52.35.Py 


\section{Introduction}

The full space $\mathbf{Z} \equiv\left\{Z_{j=1, . . N_{z}}\right\}$ of independent variables defining the shape of a stellarator configuration is large. To find attractive design points in this space, or to understand operational flexibility about a given design point, one needs insight into the topography in $\mathbf{Z}$-space of various physics figures of merit $\mathbf{P}=\left\{P_{i}(\mathbf{Z})\right\}\left(i=1, \ldots M_{p}\right)$ which characterize the machine performance $($ e.g. transport, kink stability, etc.).

An important new means for stellarator design made possible by advances in physics codes and computational power is the use of automated optimizers. For example, the NCSX design group has made extensive use of an optimizer in developing candidate configurations for an attractive QA stellarator (QAS) design. ${ }^{1}$ The optimizer conducts a search in a Z-space describing the stellarator boundary, using an objective function $F(\mathbf{P})$ which is a function of the $P_{i}$ of the configuration. While a powerful tool, the optimizer is searching a large space whose topography has been essentially unknown, and there is limited understanding of why the optimizer arrives at the design points $\mathbf{Z}_{0}$ it does. Deeper insight into this would enhance our ability to locate attractive design points (e.g., by reducing the dimensionality of the $\mathbf{Z}$-space, or by recognizing topographical features which point to superior 'valleys' of $F(\mathbf{P})$ in the $\mathbf{Z}$ space), and to examine operational flexibility about those points. Such insight can be used both to better focus the operation of an optimizer, as well as to enhance human understanding of the configurations being studied.

The control matrix (CM) approach discussed here helps provide this insight. The approach we discuss has both local and global aspects. As narrowly defined, 
the $\mathrm{CM}$ is simply the matrix $C_{i j} \equiv \partial P_{i} / \partial Z_{j}$ of first derivatives at any point $\mathbf{Z}_{0}$ in Z space, and so can give topographical information only locally. One may provide more information by expanding to second and higher orders, and most globally, one may investigate the full nonlinear variation over ranges where a power-series expansion is not practical. But even to compute the derivatives needed for $C_{i j}$ correctly, one needs to know the characteristic scales on which the the $P_{i}$ vary in $\mathbf{Z}$, so more global knowledge is important even for the local problem. And as already indicated, the more global information may also be important in locating genuinely different stellarator optima. Thus, this paper addresses both the more local and more global sides of this topographical exploration.

In Sec. II we describe the mathematical basics of the CM method, and discuss the means we use to reduce the dimensionality of $\mathbf{Z}$ space. Implementing the $\mathrm{CM}$ procedure requires exploring the scales of variation of the $P_{i}$ in $\mathbf{Z}$ in the vicinity of a design point $\mathbf{Z}_{0}$, for which we choose "C10", a candidate NCSX configuration. This is done in Sec. III. Our topographical study here finds that within an appreciable domain (variations in the $Z_{j}$ of order $1 \mathrm{~cm}$ ) about $\mathrm{C} 10$, the $P_{i}$ may be well approximated by simple quadratic expressions, and in addition, we are able to reduce the dimensionality of the $\mathbf{Z}$ space we need consider from an initial $N_{z}=78$ to 8 . As a result, in Sec. IV we apply the machinery of the local CM analysis to a greatly reduced parameter space, and within that space can compute quantities of interest using analytically tractable quadratic expressions for the $P_{i}$. We then provide the 'proof of principle' of the CM method, demonstrating that the CM mathematics correctly produces perturbations $\boldsymbol{\xi}^{i}$ with which we can independently vary the $P_{i}$, and 'nullspace' perturbations $\mathbf{v}^{i}$ which produce different configurations, but with unchanged values of the $P_{i}$. We discuss some of the fea- 
tures of these perturbations. In Sec. V we move to a more global exploration of $\mathrm{Z}$ space, applying some of the same machinery used in earlier sections to study the variation of the $P_{i}$ en route to candidate QAS configurations other than C10. Sec. VI summarizes the findings of the earlier sections, and discusses applications of the CM approach now in progress or planned for the near future.

\section{Formulation}

A single stellarator configuration may be described by a set of Fourier amplitudes, $\mathbf{X}=\left\{X_{j=1, \cdots, N_{x}}\right\} \equiv\left(R_{\mathbf{n}_{1}}, Z_{\mathbf{n}_{1}}, R_{\mathbf{n}_{2}}, \cdots, Z_{\mathbf{n}_{N_{x} / 2}}\right)$ which define the plasma boundary $[R(\theta, \zeta), Z(\theta, \zeta)]$. Here, $\mathbf{n} \equiv\left(\tilde{n}=n / N_{p}, m\right)$ are toroidal and poloidal modenumbers per period, with $N_{p}$ equal to the number of field periods. For C10 and C82, two candidate NCSX configurations ${ }^{2}$ we shall consider in this paper, the number of Fourier amplitudes is $N_{x}=78$ corresponding to maximum modenumbers of $\tilde{n}_{\max }=3$, and $m_{\max }=5$. PG1, a third QAS configuration ${ }^{3}$ discussed in Sec. V, has $N_{x}=32$. Fig. 1 shows poloidal cross sections of the plasma boundary for these three configurations.

Various measures of stellarator transport and stability are used as figures of merit in the cost function of the full configuration optimizer. ${ }^{1}$ The evaluation of all figures of merit require the calculation of an MHD equilibrium, performed using the $\mathrm{VMEC}^{4}$ code. To evaluate the transport, the magnetic fields output by VMEC in a non-straight coordinate system are re-expressed in terms of the Boozer coordinate system using the JMC code. ${ }^{5}$ The various measures of transport used by the optimizer are then easily expressed in terms of the Fourier components, $B_{m n}$, of the magnitude of the magnetic field. For stability, both internal ballooning 
and free-boundary kink modes are evaluated using the TERPSICHORE code. ${ }^{6}$

In this paper, we use the same suite of codes employed by the optimizer, and compute $M \equiv M_{p}=5$ figures of merit $\mathbf{P}=\left(\chi_{1}^{2}, \chi_{2}^{2}, W_{1}, W_{2}, \lambda\right) . P_{1-4}$ are 4 measures of the ripple strength, and hence the level of nonaxisymmetric transport one may expect, and $P_{5}$ is a measure of stability. More specifically, $\chi_{1,2}^{2} \equiv \chi^{2}\left(s_{1,2}\right)$, where $\chi^{2}(s)=\sum_{m, n \neq 0} B_{m n}^{2} / B_{00}^{2}$ and $s_{1,2}$ label two magnetic surfaces within the plasma volume containing a fixed amount of toroidal flux. The selected values of $s$ (normalized to unity at the plasma edge) are $s_{1}=0.5$, and $s_{2}=0.71$. $W_{1,2} \equiv W\left(s_{1,2}\right)$ is the "water function' ${ }^{7}$ at $s_{1,2}$, measuring the average ripple-well depth along a field line. The fifth figure of merit is $P_{5} \equiv \lambda=\omega^{2}$, the most unstable kink eigenvalue computed by TERPSICHORE (negative for unstable modes). We consider four QA-associated figures of merit for purposes of comparison among them. A single one may be used for purposes of configuration analysis, and we shall do so at appropriate points in the paper. Other figures of merit might be usefully added to the present set, such as ballooning growth rate, surface quality, or coil complexity, and the same formal machinery employed to study any such set.

$N_{x}=78$ is a large space to search, and one important objective here is to reduce this number to a more manageable value. That is, we seek a 'reduced space' $\mathbf{Z}$ contained in $\mathbf{X}$, with dimensionality $N \equiv N_{z} \leq N_{x}$ which is as small as possible while retaining the most important physics. We discuss means by which this can be done in Sec. II B. We think of Z as the space of 'control knobs' at our disposal, to which the $\mathrm{CM}$ machinery is applied, and thus its exact relation to the concrete specification of $\mathbf{X}$ already given may change, depending on application. For example, in Sec. VI we take $\mathbf{Z}$ to be a set of amplitudes describing the coil currents, for considerations of coil design or operational flexibility. However, 
for most of this paper, $\mathbf{Z}$ will be a linear subspace of $\mathbf{X}$, given by amplitudes specifying the plasma boundary.

\section{A. Control Matrix Basics}

In the reduced space, expanding $\mathbf{P}\left(\mathbf{Z}=\mathbf{Z}_{0}+\mathbf{z}\right)=\mathbf{P}\left(\mathbf{Z}_{0}\right)+\mathbf{p}$ about $\mathbf{Z}=$ $\mathrm{Z}_{0}$, one has (writing in component-form, with summation over repeated indices assumed, unless otherwise indicated)

$$
p_{i}\left(\mathbf{Z}_{0}+\mathbf{z}\right)=C_{i j}\left(\mathbf{Z}_{0}\right) z_{j}+\frac{1}{2} H_{i j k}\left(\mathbf{Z}_{0}\right) z_{j} z_{k}+(\text { h.o. }),
$$

with linear coefficients given by the $M \times N$ 'control matrix' $C_{i j}$, quadratic coefficients given by the 'Hessian' $H_{i j k}$, and h.o. $\equiv$ higher-order terms. For small enough $\mathrm{z}$ that only linear terms are needed, one has the matrix equation

$$
\mathrm{p}=\mathrm{C}_{0} \cdot \mathrm{z}
$$

with $\mathrm{C}_{0} \equiv \mathrm{C}\left(\mathbf{Z}_{0}\right)$ the control matrix at design point $\mathbf{Z}_{0}$. This may be inverted, using the Singular Value Decomposition (SVD) theorem ${ }^{8}$

$$
\mathbf{C}_{M \times N}=\mathbf{U}_{M \times N} \cdot \mathbf{\Sigma}_{N \times N} \cdot \mathbf{V}_{N \times N}^{T},
$$

with $\mathbf{U}, \mathbf{V}$ unitary matrices, and $\Sigma$ a diagonal matrix whose diagonal elements $\sigma_{i}$ are the 'singular values' of the problem. This theorem permits one to obtain a 'pseudo-inverse' $\mathrm{C}^{+}$of the nonsquare matrix $\mathbf{C}$, and provides bases spanning its range and nullspace.

Taking the particular basis set $\boldsymbol{\pi}^{i=1, M}$ in the target $\mathbf{P}$-space to be the set of unit vectors with 1 in the $i^{t h}$ position and 0 elsewhere, one has the corresponding set $\boldsymbol{\xi}^{i}$ of displacements in $\mathbf{Z}$-space

$$
\boldsymbol{\xi}^{i} \equiv \mathbf{C}_{0}^{+} \cdot \boldsymbol{\pi}^{i}
$$


where $\mathrm{C}_{0} \equiv \mathbf{C}\left(\mathbf{Z}_{0}\right)$. The $\boldsymbol{\xi}^{i}$ physically represent displacements which change a single physics parameter $P_{i}$ by unity, leaving the others unchanged. These span the range of $\mathbf{C}$. The $(N-M)$ vectors $\mathbf{v}^{i}(i=M+1, \ldots N)$ spanning the nullspace of $\mathbf{C}$ (which change the configuration without modifying any of the $P_{i}$ ) are the orthonormal set formed by those columns of $\mathbf{V}$ with $i$ such that $\sigma_{i}=0$. These are also important, permitting one to find different stellarator boundaries which have the same physics performance, giving flexibility for other criteria, e.g., improved coil design. Together, $\boldsymbol{\xi}^{i=1, . . M}$ and $\mathbf{v}^{i=M+1, . . N} \operatorname{span} \mathbf{Z}$.

For somewhat larger $\mathrm{z}$, but still small enough that only up to $H_{i j k}$ need be kept in Eq.(1), one can compute the control matrix for any $\mathbf{Z}$ in this region, via

$$
C_{i j}(\mathbf{Z}) \equiv \partial p_{i}(\mathbf{Z}) / \partial z_{j}=C_{i j}\left(\mathbf{Z}_{0}\right)+H_{i j k} z_{k}
$$

and from this, find the correct $\boldsymbol{\xi}^{i}$ at any $\mathbf{Z}$, along with simple linear expressions for the extrema $\mathbf{z}^{i}$ of the $P_{i}$, etc. We shall find that this 'quadratic model' is valid in an appreciable neighborhood about $\mathrm{C} 10$, and shall explicitly compute and make use of $C_{i j}$ and $H_{i j k}$ in Sec. III.

The $\mathbf{z}^{i}$ are easily solved for using Eq.(5). For any $i$, we may write $H_{i j k}$ in matrix notation as $\mathbf{H}_{i}$, which is a square, symmetric, $N \times N$ matrix, invertible by standard means. Then from Eq.(5), one has

$$
\mathbf{z}^{i}=-\mathbf{H}_{i}^{-1} \cdot \mathbf{c}_{0}^{i}
$$

where vector $\mathbf{c}_{0}^{i}$ is the $i^{\text {th }}$ row of matrix $\mathbf{C}_{0}$, and no summation over $i$ is implied. Similarly, taking our objective function to be a linear combination of the $P_{i}$ with weights $w_{i}, F(\mathbf{P})=w_{i} P_{i}$ (summation implied), one can readily solve for the extremum $\mathbf{z}^{F}$ of $F(\mathbf{P})$ :

$$
\mathbf{z}^{F}=-\mathbf{H}_{F}^{-1} \cdot \mathbf{c}_{0}^{F},
$$


with $\mathbf{H}_{F} \equiv w_{i} \mathbf{H}_{i}$ and $\mathbf{c}_{0}^{F} \equiv w_{i} \mathbf{c}_{0}^{i}$. Expression (7) thus gives the design point sought by an optimizer. However, as for Eq.(6), for it to be correct, $\mathbf{z}^{F}$ must fall within the domain of validity of the quadratic model for the $P_{i}(\mathbf{Z})$. An optimizer using a steepest-descent method would step opposite the direction of the $\mathbf{Z}$-space gradient $\partial_{\mathbf{Z}} F \equiv \partial F / \partial Z_{j}$. For the chosen form for $F$, this is $\partial F / \partial Z_{j}=$ $\left(\partial F / \partial P_{i}\right)\left(\partial P_{i} / \partial Z_{j}\right)=w_{i} C_{i j} \equiv w_{i}\left(\mathbf{c}^{i}\right)_{j} \equiv\left(\mathbf{c}^{F}\right)_{j}$. Using this with (5) gives

$$
\partial_{\mathbf{z}} F=\mathbf{c}^{F}=\mathbf{c}_{0}^{F}+\mathbf{H}_{F} \cdot \mathbf{z}
$$

From this and Eq.(7), one sees that at $\mathrm{z}=\mathrm{z}^{F}$ one has $\partial_{\mathrm{z}} F=0$, as one expects. Additionally, using Eqs. (4) and (8), one finds that the component of $\boldsymbol{\xi}^{i}$ in the direction of this gradient is $\partial_{\mathbf{Z}} F \cdot \boldsymbol{\xi}^{i}=w_{i}$.

The $P_{i}(\mathbf{Z})$ provide $M$ coordinates of a special global coordinate system on $\mathrm{Z}$-space, chosen to characterize the physics performance of the stellarator. One may imagine supplementing these by a further set $\left\{Q_{i}(\mathbf{Z})\right\},(i=M+1, . . N)$ of functionally independent quantities to fully parametrize $\mathbf{Z}$, also chosen on the basis of their physical relevance or independence from stellarator performance. Obtaining the (nonlinear) transformation between the control knobs $Z_{j}$ and the performance parameters $P_{i}$ and $Q_{i}$ lies at the core of stellarator design. At each point $\mathbf{Z}$, the basis set $\left\{\boldsymbol{\xi}^{i=1, . . M}, \mathbf{v}^{i=M+1, . . N}\right\}$ coming from the CM formulae introduced here provides a local description of this performance-based coordinate system, with the $\boldsymbol{\xi}^{i}$ performing a role akin to that of the reciprocal basis vectors $\mathbf{e}_{i}$ of the contravariant representation $A^{i} \mathbf{e}_{i}$ of any vector $\mathbf{A}$, pointing in a direction normal to the gradients of all $P_{i}$ but that with the specified $i$. 


\section{B. Reducing the dimensionality of $\mathrm{Z}$}

The CM framework just described may be applied to the full $N_{x}=78$ dimension space $\mathbf{X}$, or to any subspace $\mathbf{Z}$ of that space. Before applying the method in the vicinity of configuration $\mathrm{C} 10$, it is useful to begin reducing the dimensionality $N_{z}$ of $\mathbf{Z}$. In this paper, we shall use 2 methods to accomplish reductions:

(a) First, as pointed out by Hirshman and Breslau, ${ }^{10}$ there is a redundancy in the $\mathrm{X}$-specification, with many perturbations $\mathrm{x}$ of $\mathrm{X}$ modifying the representation of the boundary, rather than the boundary shape itself. The equivalent nonredundant representation we present in this section produces a reduction of a factor of 2 , yielding a nonredundant subspace $\mathbf{Y} \subset \mathbf{X}$ of dimension $N_{y}=39$ for the C10C82 family.

(b) In Sec. III, we further reduce $N_{z}$ from 39 to 8 , by selecting only the perturbations most effective in varying the $P_{i}$ of interest, resulting in an approximate 'reduced-model' description of configuration space.

Regarding reduction (a), independent variations in $R_{m n}$ and $Z_{m n}$ produce not just changes in the physical shape of the plasma boundary, but also changes in the poloidal angle variable, which is not uniquely defined. A nonredundant boundary deformation is made by instead prescribing the linear combinations of the $R_{m n}, Z_{m n}$ that define normal displacements (at each $\phi$ plane) to the plasma boundary. For a plasma boundary in real space $\vec{X}$ defined by

$$
\vec{X}(\theta, \phi)=R(\theta, \phi) \hat{R}(\phi)+Z(\theta, \phi) \hat{Z}
$$

a general displacement is $\vec{Y}=\delta \vec{X}=\delta R \hat{R}+\delta Z \hat{Z}$, and a normal displacement is $^{11}$

$$
\vec{Y} \cdot \frac{\partial \vec{X}}{\partial \theta} \times \frac{\partial \vec{X}}{\partial \phi}=R\left(\frac{\partial R}{\partial \theta} \delta Z-\frac{\partial Z}{\partial \theta} \delta R\right)
$$


Multiplying by $\cos (m \theta+n \phi)$ and integrating over $\theta$ and $\phi$ yields a matrix equation in the form

$$
Y_{i}=\sum_{j} B_{i j} X_{j}
$$

Here, as earlier, $\mathbf{X} \equiv\left\{X_{j=1, \ldots N_{x}}\right\}$ is the set of Fourier expansion coefficients of

both $\delta R$ and $\delta Z$, while $\mathbf{Y} \equiv\left\{Y_{i=1, \ldots N_{y}}\right\}$ is the set of Fourier coefficients of the normal displacement to the plasma boundary, and $B_{i j}$ is the $N_{y} \times N_{x}$ rectangular influence matrix that relates the two. To compute the $X_{j}$ required by VMEC in terms of the $Y_{i}$, we employ the same SVD decomposition as in Eq.(3) to invert the matrix $B_{i j}$. In Fig. 2 is shown the deformation produced by a typical $Y_{i}$, for $\mathbf{n}_{i}=(1,0)$, plotted in poloidal cross-section at several values of $\tilde{\zeta} \equiv N_{p} \zeta$ across a period of the machine.

Before we can apply the approximate reduction method (b) or take the numerical derivatives needed to carry out the CM prescription, we need to ascertain the scales of variation of the $P_{i}$ in the $\mathbf{X}$ or $\mathbf{Y}$ space. This is done in the following section.

\section{Topography of Z-space near C10}

The validity of Eqs. (1) or (2) depends on the typical scales of variation in $\mathrm{Z}$-space of the $P_{i}$, which previously have been largely unknown. In the vicinity of the C10 family of configurations, we have assessed this variation for the $P_{i}$ presently being used for all $78 X_{j}$ as well as for the nonredundant amplitudes $Y_{j}$.

Some typical results in the space $\mathbf{Z}=\mathbf{Y}$ are shown in Fig. 3, showing the variation of the $P_{i}$ computed from VMEC and TERPSICHORE with deformation 
amplitude $z_{j} \equiv \delta Z_{j}$ (in meters) of the given $Z_{j}=Y_{j}$. Four harmonics $\mathbf{n}_{j}$ are shown. The top two are for $\mathbf{n}=\{(1,0),(-3,1)\}$, to which the QA measure $P_{1}$ is sensitive, and the bottom two are for $\mathbf{n}=\{(1,3),(2,4)\}$, to which the kink eigenvalue $P_{5}$ is relatively sensitive. The size of the domain shown $\left(\Delta Z_{j}= \pm .01\right.$ $\mathrm{m}$ ) is appreciable, large enough to encompass C82 as well as C10 (see Sec. VA).

Probably because of the symmetrizing action of the optimizer in creating C10, most directions $Z_{j}$ resemble the top pair: the fractional variation $P / P_{0}$ of $P_{1}\left(Z_{j}\right)$ is much larger than that for $P_{5}$ (reflecting the near-optimal value of the unperturbed $P_{10}$ in the denominator), and $P_{1}$ is a parabolic curve, with vertex often near $z_{j}=0$. For all $Z_{j}$, the variation of both $P_{i}$ is smooth and rather unstructured, approximable by the quadratic expansion (1) over all or most of this domain.

Given this knowledge, in Fig. 4 are shown histograms of the fractional sensitivity $\left(P_{i} / P_{i 0}-1\right)$ over the $(\tilde{n}, m)$-plane (hence showing all $\left.Z_{j}\right)$, for $i=1-3$ and 5 , and for a fixed value $d Z=.002 \mathrm{~m}$ for each $z_{j}$, a modest fraction of the $Z_{j}$ scale length just shown in the plots of Fig. 3. (The $i=4$ histogram is not shown simply to conserve space.)

Though the QA measures $P_{i=1-4}$ are linearly independent, their histograms are quite similar, and these are markedly different from that for the kink measure $P_{5}$.

With the information in the sensitivity histograms, we can apply reduction method (b) introduced in Sec. II. We rank the harmonics, selecting the 4 to which $P_{1}$ is most sensitive, and the 4 to which $P_{5}$ is most sensitive, resulting in a final reduced model with $N_{z}=8$ harmonics. We have chosen a single one of the 4 QAassociated $P_{i}$ as representative of QA measures, confirmed from the similarity of the curves for $P_{i=1-4}$ in Fig. 3. The choice of 4 harmonics for each is somewhat 
arbitrary, chosen to produce a relatively simple system on which to develop the CM machinery, yet rich enough to display the control flexibility we are seeking. We have examined two $N_{z}=8$ reduced models, whose difference comes from somewhat different ranking criteria. The models were found to possess similar properties. The criterion for the model presented here is simply taking the harmonics with the largest values of $\left|P_{i} / P_{i 0}-1\right|$. The 8 harmonics of this model are accordingly $\mathbf{n}_{j}=\{(1,0),(2,0),(3,0),(-3,1) ;(1,3),(1,4),(2,4),(1,5)\}$,

with the first 4 most affecting $P_{1}$, and the last 4 most affecting $P_{5}$. One notes that the $P_{1}$ set (affecting QA-ness) have small $m$ and a range of $\tilde{n}$, while the $P_{5}$ set have $\tilde{n} \sim 1$ or 2 and a range of $m$. The top 2 plots of Fig. 3 are seen to belong to the $P_{1}$ set, and the bottom 2 to the $P_{5}$ set.

\section{CM Analysis of the Reduced Model}

Having now established the scales of variation in $\mathrm{Y}$-space, and selected from sensitivity histograms the $Y_{j}$ comprising our reduced model, we are in a position to evaluate the CM tensors $C_{i j}$ and $H_{i j k}$ and from these the vectors $\mathbf{z}^{i}, \boldsymbol{\xi}^{i}$, and $\mathbf{v}^{i}$ introduced in Sec. II, and to demonstrate that these have the intended properties described in that section. In addition to the base configuration $\mathbf{Z}_{0}$, which we take to be $\mathrm{C} 10,2 N_{z}^{2}$ perturbed points $\mathbf{Z}$ must be evaluated for computing the $H_{i j k}$, including $2 N_{z}$ points also needed for the $C_{i j}$. For $N_{z}=8$, this gives $2 N_{z}^{2}=128$ points to which VMEC, JMC, and TERPSICHORE must be applied, an appreciable but manageable computational task. 


\section{A. Quadratic Model}

Performing the evaluation of $C_{i j}$ and $H_{i j k}$, in Fig. 5 we provide a first check that the resultant quadratic model of the $P_{i}(\mathbf{Z})$ is behaving as it should, plotting $P_{1}$ and $P_{5}$ versus the same $4 Z_{j}$ as shown in the numerical results of Fig. 3. One notes the good agreement. The increment for the required first and second derivatives was $d Z=.002 \mathrm{~m}$, which predicts well the values of the $P_{i}$ for displacements over the range $\Delta Z=.02 \mathrm{~m}$ shown in Fig. 3 or 5 . Even for $\mathbf{n}=(1,3)$, where $P_{5}$ in Fig. 3 cannot be approximated by a quadratic over the full $z_{j}$ range shown, the quadratic model does well over about the half of the full range near $\mathrm{C} 10\left(z_{j}=0\right)$.

Using $C_{i j}$ and $H_{i j k}$, one readily computes the $\mathrm{z}^{i}$ from Eq.(6). One finds, for example $\mathbf{z}^{1}=\{0.15,-0.38,-0.20,-0.14,-2.11,-14.5,2.29,7.66\} \times 10^{-3}$, and $\mathrm{z}^{5}=\{-5.35,1.61,0.19,0.26,0.33,-3.39,4.97,-4.48\} \times 10^{-3}$ (meters).

In Fig. 6 we visualize the topography in the vicinity of C10, with contour plots of $P_{1}$ (left) and $P_{5}$ (right) from the quadratic model over a plane of 2 chosen $z_{j}$. The top plots are for 2 QA-related $z_{j}$, and the contours for these are elliptical. The bottom plots are for $1 \mathrm{QA}-$ related and 1 kink-related $z_{j}$, and one notes here that $P_{1}$ is almost independent of $z_{8}$, while $P_{5}$ is almost independent of $z_{1}$. It is somewhat fortuitous that this property is nearly obeyed for individual $z_{j}$; it need be exactly obeyed only for variations in the $\xi^{1}$ and $\xi^{5}$ directions.

Applying the SVD-algorithm to invert $C_{i j}$, we use Eq.(4) to compute the $\boldsymbol{\xi}^{i}$ and $\mathbf{V}$ from Eq.(3) in obtaining $\mathbf{v}^{i}$. As opposed to the $\mathbf{z}^{i}$, the values of these depend on the how many of the $P_{i}$ one chooses to keep in $C_{i j}$. One may keep only $P_{1}$ and $P_{5}$, yielding an $M_{p}=2$ problem with a nullspace of $N_{z}-M_{p}=8-2=6 \mathbf{v}^{i}$, , or solve the more-constrained problem increasing $M_{p}$ (up to 5, here). Here, we consider the $M_{p}=2$ problem, obtaining $\boldsymbol{\xi}^{1}=\{-3.92,9.82,5.99,-1.19,0.29,0.55$, 
$-0.56,0.22\}$, and $\xi^{5}=\{0.09,-0.78,0.03,-0.11,2.77,2.05,-2.53,-4.10\}$. One notes that the first 4 components of $\boldsymbol{\xi}^{1}$ (the QA-related $z_{j}$ ) are dominant, while the last 4 components dominate in $\xi^{5}$. While this might be expected, it is not necessary: an independence of (e.g.) $P_{1}$ from $\xi^{5}$ could arise from a cancellation of the effects of appreciable components in $\xi^{5}$ of the QA-related $z_{j}$.

\section{B. 'Proof of Principle' of the CM method}

Having obtained the $\boldsymbol{\xi}^{i}$ and $\mathbf{v}^{i}$, we can now test the crucial properties discussed in Sec. II of these directions in $\mathrm{Z}$ space, viz., showing that the boundary perturbations the $\boldsymbol{\xi}^{i}$ describe actually permit independent control of the $P_{i}$, and that those of the $\mathrm{v}^{i}$ actually leave the $P_{i}$ unchanged. In this section we provide this key demonstration, and examine some of the features of the deformations these vectors produce.

The demonstration needed is agreement between the analytically-expected variation of the $P_{i}$ obtained from the quadratic model in the direction of a $\boldsymbol{\xi}^{i}$ or $\mathbf{v}^{i}$, and the variation numerically-obtained from a sequence of equilibria perturbed from $\mathrm{C} 10$ in that direction. This comparison is provided in Fig. 7 for perturbations $\alpha \boldsymbol{\xi}^{1}$ (left) and $\alpha \boldsymbol{\xi}^{5}$ (right), and in Fig. 8 for perturbations $\alpha \mathbf{v}^{3}$ (left) and $\alpha \mathbf{v}^{8}$ (right). Here, $\alpha$ is a scaling parameter, with value specified on the horizontal axis. The analytic expections are on the top row, and the numerical results on the bottom row. One notes that the variations are as expected. Perturbations in the $\boldsymbol{\xi}^{1}$ direction do in fact vary $P_{1}$ while leaving $P_{5}$ unchanged, and similary for $\boldsymbol{\xi}^{5}$. For the $\xi^{5}$ perturbation, there is somewhat more wobble visible in the $P_{1}$ curve than for the $P_{5}$ curve for the $\xi^{1}$ perturbation, because of the greater sensitivity of $P_{1}$ to most $z_{j}$. Keeping $P_{1}$ constant therefore requires a more delicate balance of the 
harmonics contributing in $\boldsymbol{\xi}^{5}$.

We visualize these perturbations in Figs. 9-10. Fig. 9 shows contour and surface plots over the $(\theta, \tilde{\zeta})$-plane of perpendicular displacements $\xi_{\perp}^{1}$ and $\xi_{\perp}^{5}$ (top), and $v_{\perp}^{3}$ and $v_{\perp}^{8}$ (bottom). $\xi_{\perp}^{5}$ is seen to vary more rapidly with $\theta$ and less rapidly with $\tilde{\zeta}$ than $\xi_{\perp}^{1}$, consistent with the harmonic contributions in the sensitivity histograms in Fig. 4. The effect of these perturbations on the boundary are shown in poloidal cross-section in Fig. 10. In particular, one notes that $\boldsymbol{\xi}^{5}$ for diminishing the kink produces an indentation of the outboard side at the half-period $\tilde{\zeta}=\pi$, enhancing the (negative) triangularity which that cross-section possesses. This is consistent with the earlier empirical observation ${ }^{1}$ that kink stability can be helped by providing such an indentation. Here, this finding emerges simply from the $\mathrm{CM}$ calculation for $\boldsymbol{\xi}^{5}$. However, one also notes that indentation at $\tilde{\zeta}=\pi$ alone is not enough to stabilize the kink: $v_{\perp}^{8}$ also causes an indentation. However, its variation

with $\tilde{\zeta}$ is markedly different from that of $\xi_{\perp}^{5}$, having an $\tilde{n}=1$ character, in contrast to the $\tilde{n}=0$ character for $\xi_{\perp}^{5}$.

\section{Global Topography: Other QAS Design Points}

C10 and C82 (see Fig. 1) were arrived at along an involved path of human interaction with the optimizer, and it is unclear that other regions of Z-space, which would have been reached from different starting points, might not yield superior configurations. Thus, in this section we initiate an exploration of regions of $\mathbf{Z}$ space further from $\mathbf{C} 10$. As guideposts to promising regions to explore, one can look near other proposed QAS configurations ${ }^{3,9}$ with the same methods. Here, we consider the variation of the $P_{i}$ as one moves from one such reference point 
$\mathrm{Z}_{0}$ to another.

\section{A. The path from $\mathrm{C10}$ to $\mathrm{C82}$}

We begin by considering the $P_{i}$ along a straight-line trajectory $\mathbf{Z}=\mathbf{Z}_{C 10}+$ $\alpha\left(\mathbf{Z}_{C 82}-\mathbf{Z}_{C 10}\right)$ connecting $\mathrm{C} 10$ with $\mathrm{C} 82$, as $\alpha$ runs from 0 to 1 . These two configurations are fairly close in Z-space. We may quantify this by introducing the simple norm: $|\mathbf{X}| \equiv\left(\sum_{j} X_{j}^{2}\right)^{1 / 2}$. With this definition, $\left|\mathbf{X}_{C 82}-\mathbf{X}_{C 10}\right| \simeq .041 \mathrm{~m}$, in

comparison with the much larger 'distance' to PG1 (see below), $\left|\mathbf{X}_{P G 1}-\mathbf{X}_{C 10}\right| \simeq$ $.228 \mathrm{~m}$

C82 was obtained from $\mathrm{C} 10$ in an effort to stabilize the kink. The level of QA-ness was slightly degraded in compensation. This is borne out by the $P_{i}$ 's along the straight-line path in Z-space, shown in Fig. 11. While the kink growth rate falls off to an acceptably low value $\left(\lambda_{C 82} / \lambda_{C 10} \simeq .05\right), P_{1}$ actually moves to a somewhat lower value (better quasisymmetry) about midway along the trajectory, and then rises at $\mathrm{C} 82$ to a value slightly larger than for $\mathrm{C} 10$. One notes that the quadratic approximation would be adequate to describe the variation of the $P_{i}$ along this trajectory.

\section{B. The path from C10 to PG1}

Configuration PG1 (see Fig. 1) is characterized by ${ }^{3}$ much better kink stability $(\lambda>0)$ than $\mathrm{C} 10$ or $\mathrm{C} 82$, but substantially worse quasisymmetry, due mainly to a large mirror field $B_{m=0, \tilde{n}=1}$ present to assure ballooning stability.

As indicated above, its separation from $\mathrm{C} 10$ in $\mathrm{Z}$-space is far greater than that of $\mathrm{C} 82$, and is generally considered to be in a quite different region of $\mathbf{Z}$. 
Nevertheless, as one sees in Figs. 12, even over this relatively large distance the $P_{i}$ do not fluctuate greatly, but instead vary smoothly, and almost monotonically, in a manner consistent with the qualitative description of the physics differences given just above between the 2 stellarators.

Applying the same tools to PG1 as described above for C10, one finds sensitivity histograms for the $\boldsymbol{\xi}^{i}$ which resemble those for C10. Again, those for $i=1-4$ are similar to each other (and to those for C10), and differ from that for $i=5 . \xi^{5}$, which reduces the kink growth rate, is found to enhance the positive triangularity which PG1 possesses in the half-period $\tilde{\zeta}=\pi$, consistent with tokamak-based intuition on kink stabilization, an effect opposite that found for $\mathrm{C} 10$, which as noted earlier has negative triangularity at $\tilde{\zeta}=\pi$.

\section{Discussion and Summary}

In the foregoing sections we have described and applied the CM approach, mostly in the vicinity of the $\mathrm{C} 10-\mathrm{C} 82$ family of stellarators to which an optimizer has led the NCSX group. For the first time, we are getting a picture of the topography of the configuration space $\mathbf{Z}$ in which the NCSX optimizer has been searching for good QA stellarators. The local CM method would be applicable and useful even in a $\mathbf{Z}$-space where the $P_{i}$ were highly involuted, but instead we find that these are rather smooth and unstructured, even over distances in $\mathbf{Z}$ generally considered large. In an appreciable neighborhood of $\mathrm{C} 10\left(\Delta Z_{j} \sim 1 \mathrm{~cm}\right)$ the $P_{i}$ may be modeled by a quadratic function of $\mathrm{z}=\mathbf{Z}-\mathbf{Z}_{0}$.

From this topographical information, we have produced a restricted configuration space which reduces the dimensionality from $N_{z}=78$ to 8 while retaining 
much of the interesting physics in the vicinity of $\mathrm{C} 10$, and within this space obtained the first and second-order coefficients $C_{i j}$ and $H_{i j k}$ describing the simple quadratic variation of the $P_{i}$. This permits one to compute quantities of interest for the $\mathrm{CM}$ formalism using analytically tractable expressions. We have demonstrated that the $\mathrm{CM}$ method produces perturbations $\boldsymbol{\xi}^{i}$ in $\mathbf{Z}$ with which one can independently vary the $P_{i}$, and perturbations $\mathbf{v}^{i}$ producing different configurations with unchanged values of $P_{i}$.

For both $\mathrm{C} 10$ and PG1, the sensitivity histograms for $i=1-4$ resemble one another, and differ from that for $i=5$. Correspondingly, the $\boldsymbol{\xi}^{i}$ for the 4 different QA-associated figures of merit $(i=1-4)$ are similar in appearance, and these differ from that for the kink $(i=5)$.

For $\mathrm{C} 10, \xi^{5}$ manifests the outboard indentation previously empirically observed to stabilize the kink, enhancing C10's negative triangularity at $N_{p} \zeta=\pi$, while for PG1, $\xi^{5}$ enhances its positive triangularity, consistent with tokamak intuition on kink stabilization.

The work discussed in this study has taken as its free 'control knobs' $Z_{j}$ displacements of the plasma boundary. However, exactly the same procedures may be used to study how a given set of coil currents described by amplitudes $\mathbf{I} \equiv\left\{I_{j}\right\}$ could produce a range of physics behavior $\mathbf{P}$, with the specialization $Z_{j} \rightarrow I_{j}$. Here, the $I_{j}$ may represent either $K_{\mathbf{n}_{j}}$, the Fourier amplitudes of the current potential $K(\theta, \zeta)$, for coil design, or $J_{j}$, the amount of current in the $j^{\text {th }}$ coil of a given coil set, to study operational flexibility. Then the $P_{i}(\mathbf{Z})$ can be computed almost as done in the present study, but using free-boundary instead of fixed-boundary VMEC.

It will also sometimes be useful to extend the $P_{i}$ and $Z_{j}$ beyond the sets spec- 
ified thus far. E.g., to study startup scenarios, the $Z_{j}=J_{j}$ could be supplemented to also include $Z_{\beta} \equiv\langle\beta\rangle$, and perhaps a parameter characterizing the peakedness of the pressure profile. Then, for example, the relative size of $Z_{\beta}$ to the other $Z_{j}$ in the $\mathbf{v}^{i}$ would specify how the coil currents should be raised as $\langle\beta\rangle$ is during startup in order not to change the QA-ness or kink stability of the machine. For coil design, the $P_{i}$ could be supplemented to include a measure of coil complexity, e.g., one already used by the NCSX group, ${ }^{12}$

$P_{6} \equiv \sum_{\mathbf{n}} m^{p+1} K_{\mathbf{n}}^{2} / \sum_{\mathbf{n}} m^{p} K_{\mathbf{n}}^{2}$, with $p=1-4$. Then applying the CM method just as in the present study, $\boldsymbol{\xi}^{6}$ would describe perturbations which would reduce the coil complexity, while maintaining the same physics performance. These and other such applications are planned for future work.

\section{Acknowledgments}

We would like to thank Allen Boozer and Long-Poe Ku for useful discussions, and Steve Hirshman and Tony Cooper for use of VMEC and TERPSICHORE. Dr. Boozer was particularly helpful with the analysis in Sec. IIB. This work supported by U.S.Department of Energy Contract No.DE-AC02-76-CHO3073. 


\section{References}

${ }^{1}$ A. Reiman, G. Fu, S. Hirshman, D. Monticello, H. Mynick, et al., European Physical Society Meeting on Controlled Fusion and Plasma Physics Research, Maastricht, the Netherlands, June 14-18, 1999, (European Physical Society, Petit-Lancy, 1999).

${ }^{2}$ G.H. Neilson, A.H. Reiman, M.C. Zarnstorfff et al., "Physics Basis for HighBeta, Low-Aspect-Ratio Stellarator Experiments" 1999 APS (to be published in Phys. of Plasmas).

${ }^{3}$ P. Garabedian, L.P. Ku, Phys. Plasmas 6, 645 (1999).

${ }^{4}$ S.P. Hirshman, W.I. van Rij and P. Merkel, Comput. Phys. Commun. 43, 143 (1986).

${ }^{5}$ J. Nuehrenberg and R. Zille, "Proceedings 5th International Workshop on Stellarators", Schloss Rinberg, 1984 (Commission of the European Communities, Brussels, 1984) EUR 9618EN 339.

${ }^{6}$ D.V. Anderson, A. Cooper, U. Schwenn and R. Gruber in Proc. of the Joint Varenna-Lausanne International Workshop on Theory of Fusion Plasmas, (Editrice Compositori, Bologna, 1988) p93.

${ }^{7}$ M.Yu. Isaev, M.I. Mikhailov, D.A. Monticello, H.E. Mynick, A.A. Subbotin, L.P. Ku, A.H. Reiman, Phys. Plasmas 63174 (1999).

${ }^{8} \mathrm{Wm}$. H. Press, et al., Numerical Recipes in Fortran 77, (Cambridge University Press, 1996), p.51ff. 
${ }^{9}$ N. Nakajima, M. Yokoyama, M. Okamoto, J. Nührenberg, Plasma Physics Reports 23, 460 (1997).

${ }^{10}$ S.P. Hirshman and J. Breslau, Phys. Plasmas 5, 2664 (1998).

${ }^{11}$ A.H. Boozer, (private communications, 1999).

${ }^{12}$ S.P. Hirshman, (private communications, 1999). 


\section{Figures}

FIG. 1. Poloidal cross-sections of the boundaries of reference QAS configurations $\mathrm{C} 10, \mathrm{C} 82$, and PG1, at $\tilde{\zeta}=0, \pi / 2$, and $\pi$.

FIG. 2. Poloidal cross-sections of C10 boundaries, unperturbed (solid curve) and perturbed by $.01 Y_{\mathbf{n}=(1,0)}$, at toroidal positions $\tilde{\zeta}=0, \pi / 2$, and $\pi$.

FIG. 3. Variation of $P_{i=1-5}$ computed from VMEC and TERPSICHORE, of equilibria with deformation amplitude $z_{j}$ (in meters), for representative harmonics $\mathbf{n}_{j}=\{(1,0),(-3,1)\}$ (top) and $\{(1,3),(2,4)\}$ (bottom).

FIG. 4. Histograms of the fractional variation $P_{i} / P_{i 0}-1$ for $i=1-3$ and 5 over the full $(\tilde{n}, m)$-plane for $\mathrm{C} 10$, for a fixed variation $d Z=.002 \mathrm{~m}$ in amplitudes $Z_{j}$.

FIG. 5. Variation of $P_{1}=\chi_{1}^{2}$ and $P_{5}=\lambda$ with deformation amplitude $z_{j}$ for the same harmonics as in Fig. 3, but computed from the quadratic approximation of Eq.(1).

FIG. 6. Contour plots of $P_{1}$ (left) and $P_{5}$ (right) over the $\left(z_{1}, z_{2}\right)$ plane (top) and $\left(z_{1}, z_{8}\right)$ plane (bottom).

FIG. 7. Comparison of the analytical (top row) versus numerical (bottom row) variation of $P_{1,5}$ for perturbations $\alpha \xi^{1}$ (left) and $\alpha \xi^{5}$ (right), confirming that these displacements provide independent control over $P_{1}$ and $P_{5}$.

FIG. 8. Comparison of the analytical (top row) versus numerical (bottom row) variation of $P_{1,5}$ for perturbations $\alpha \mathbf{v}^{3}$ (left) and $\alpha \mathbf{v}^{8}$ (right), confirming that these nullspace displacements have no effect on $P_{1}$ or $P_{5}$. 
FIG. 9. Contour and surface plots over the $(\theta, \tilde{\zeta})$-plane of perpendicular displacements $\xi_{\perp}^{1}$ and $\xi_{\perp}^{5}$ (top), and $v_{\perp}^{3}$ and $v_{\perp}^{8}$ (bottom).

FIG. 10. Poloidal cross-sections of boundaries of C10 (solid curve) and C10 perturbed by $.002 \boldsymbol{\xi}^{1,5}$ and $.01 \mathbf{v}^{3,8}$ at toroidal positions $\tilde{\zeta}=0$, and $\pi$.

FIG. 11. Plot of the fractional variation $P_{i} / P_{i 0}$ for $i=1-5$ along a straight-line path in Z -space from $\mathrm{C} 10(\alpha=0)$ to $\mathrm{C} 82(\alpha=1)$. These have an $\mathbf{X}$-space distance between them of $.041 \mathrm{~m}$.

FIG. 12. (a) Plot of the fractional variation $P_{i} / P_{i 0}$ for $i=1-5$ along a straightline path in Z-space from $\mathrm{C} 10(\alpha=0)$ to PG1 $(\alpha=1)$. These have an $\mathrm{X}$-space distance between them of $.228 \mathrm{~m}$. (b) As (a), but with blowup of vertical scale, to show more clearly the variation in $P_{5}$. 

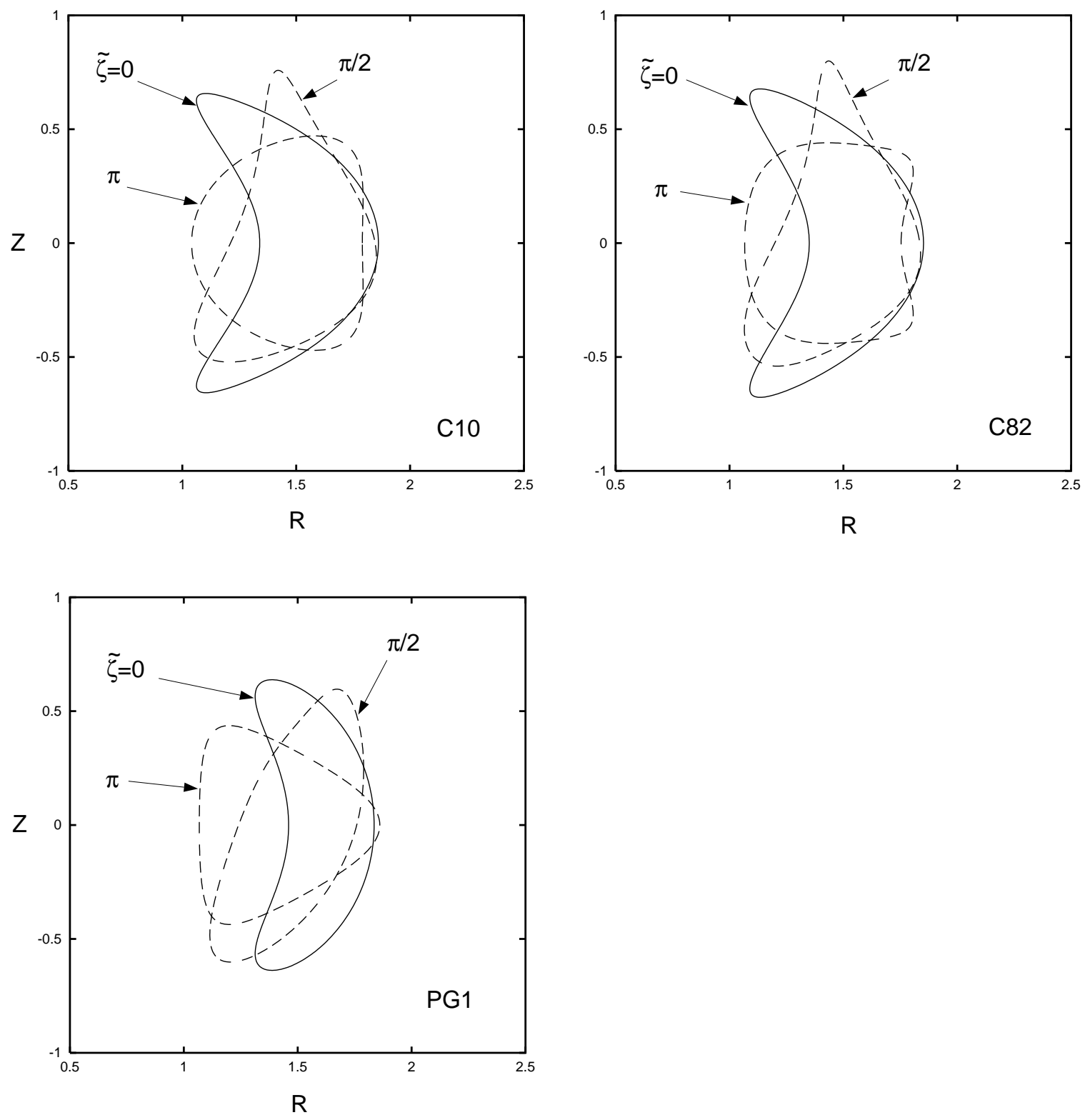

Figure 1: 

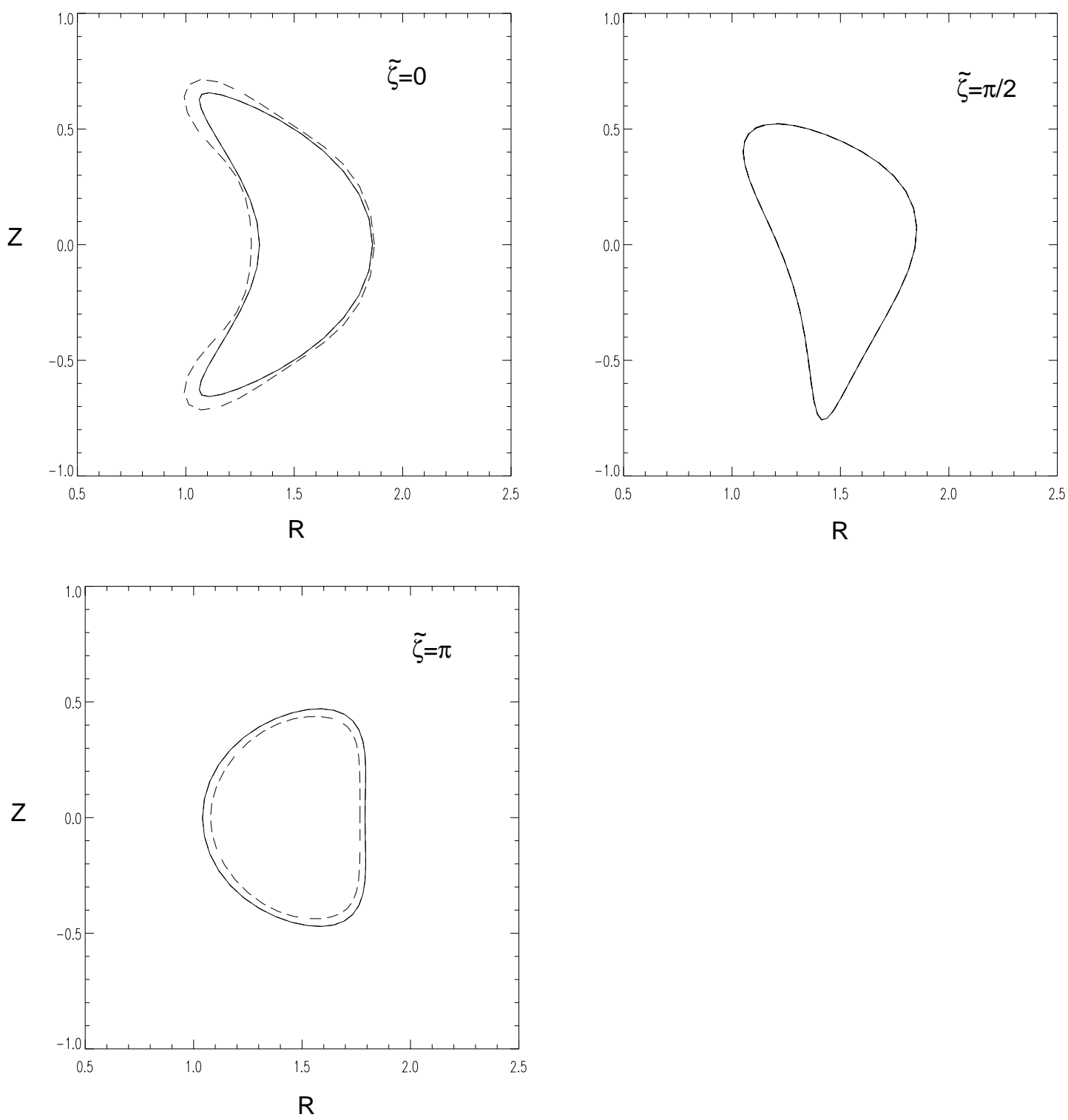

Figure 2: 

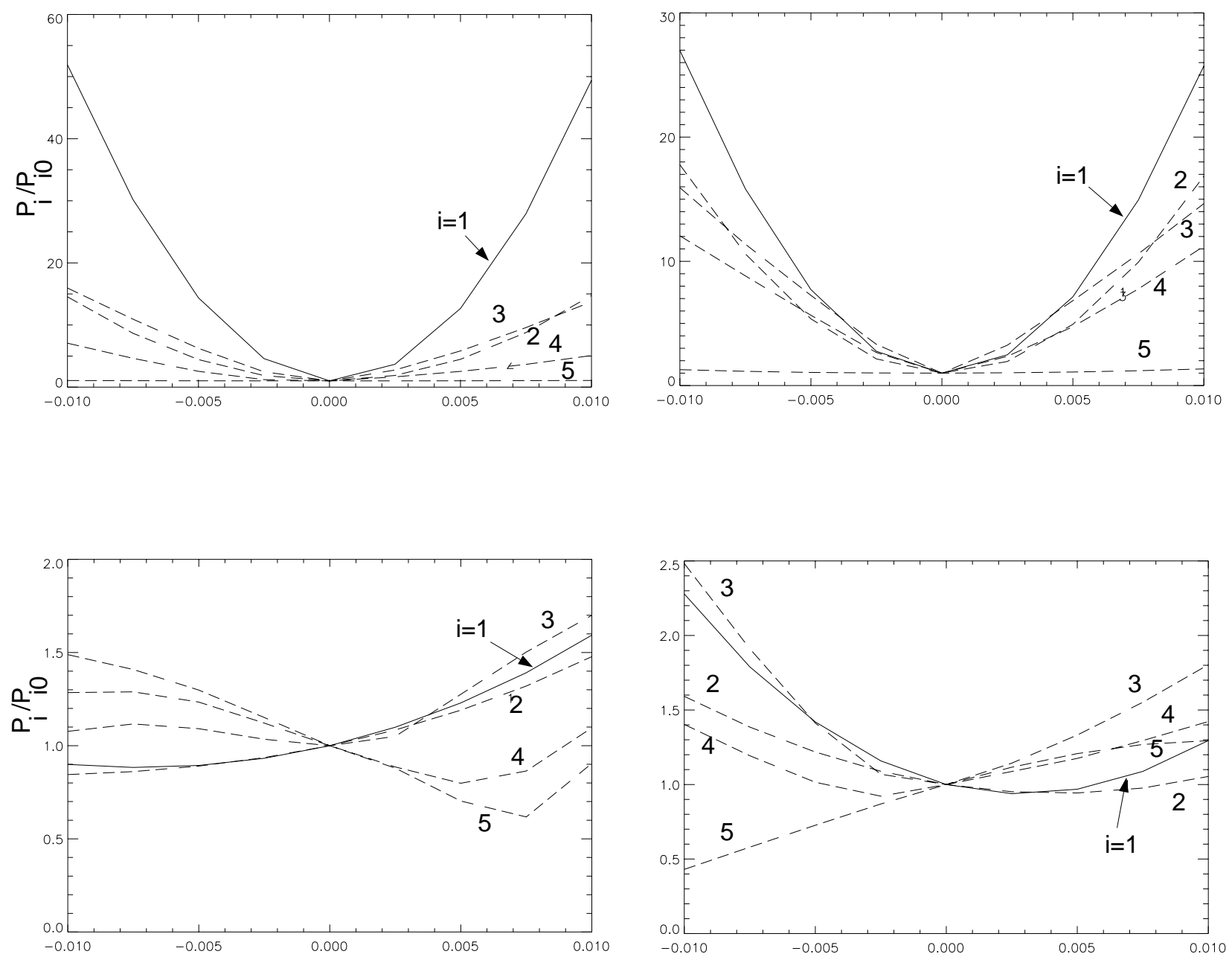

Figure 3: 


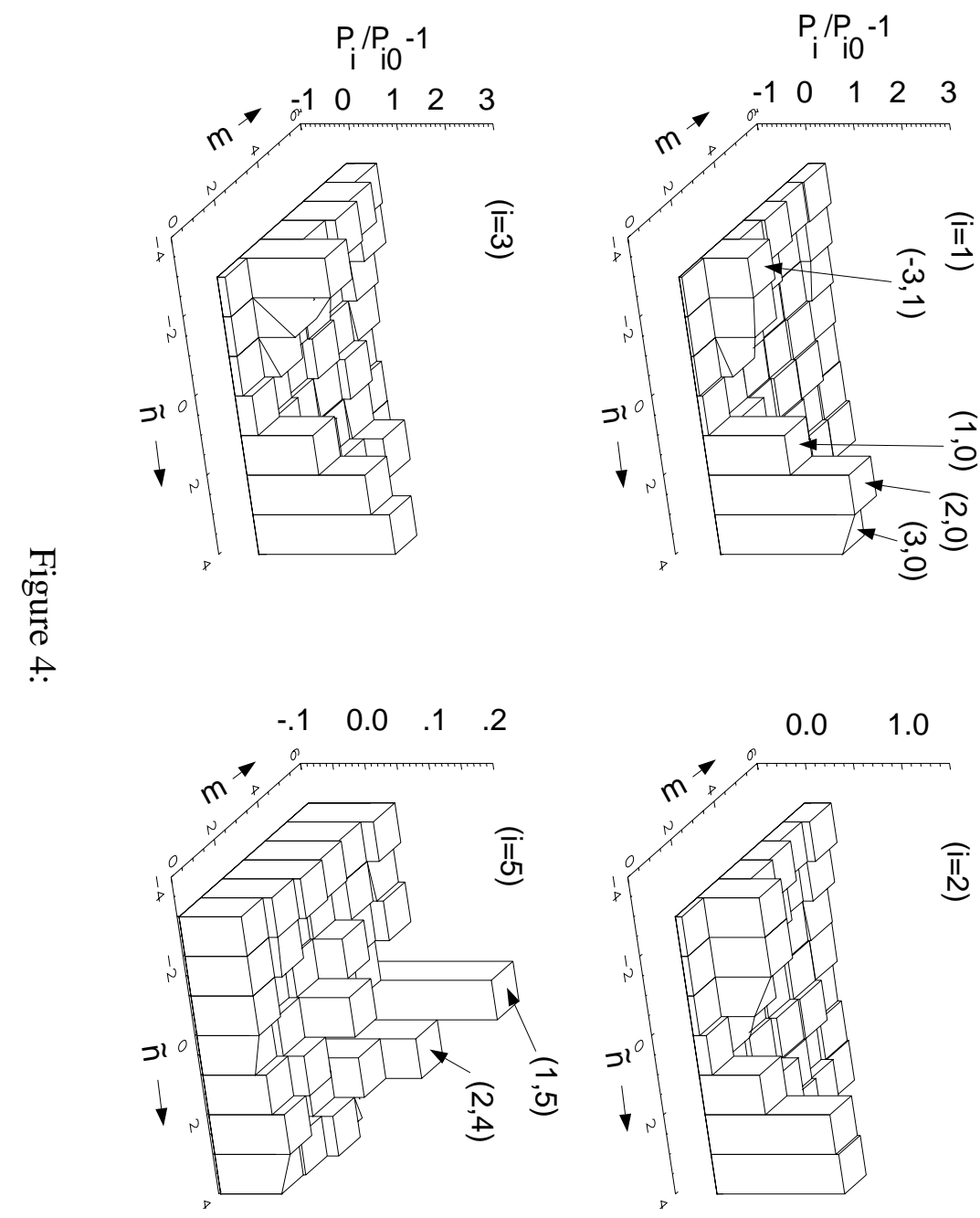



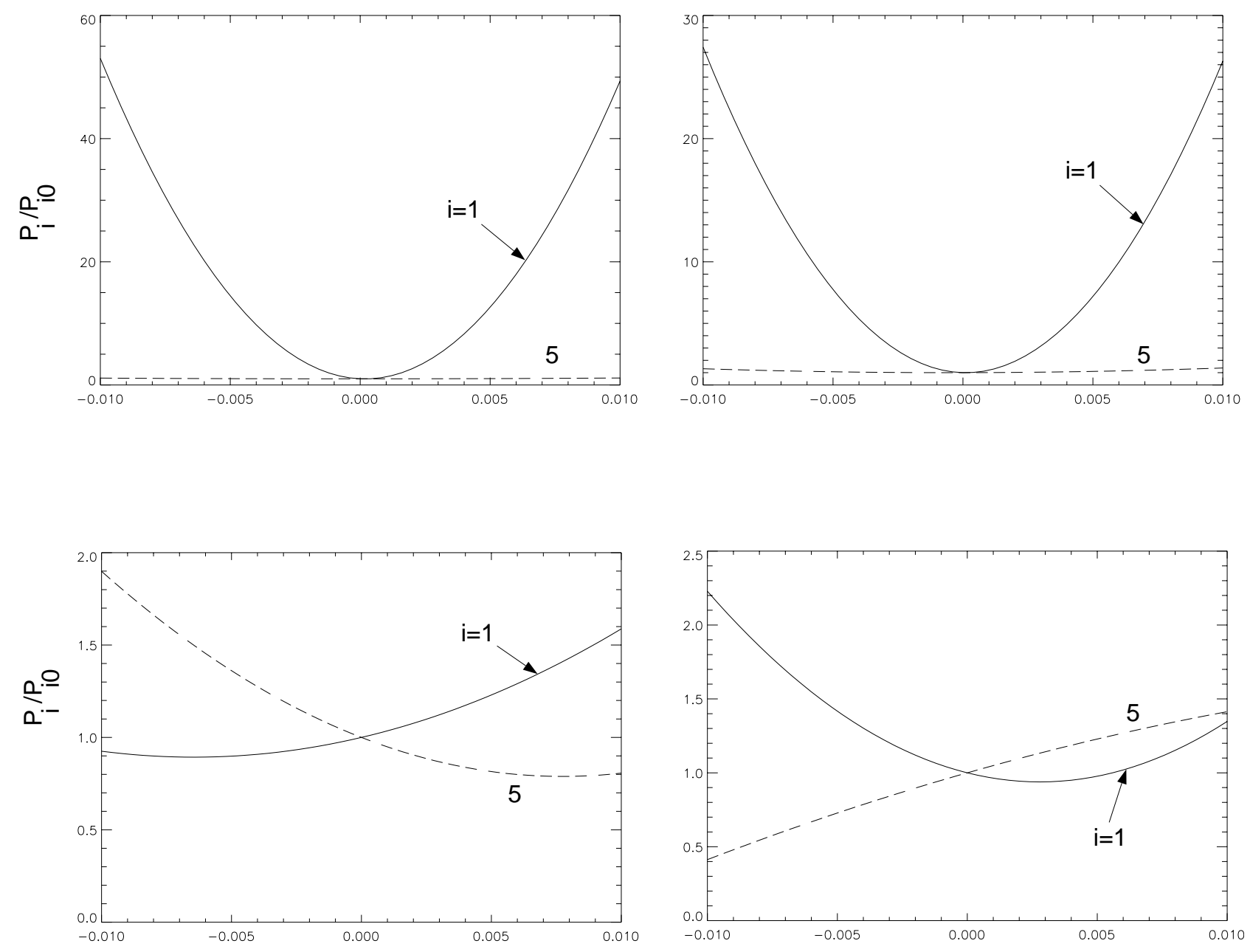

Figure 5: 

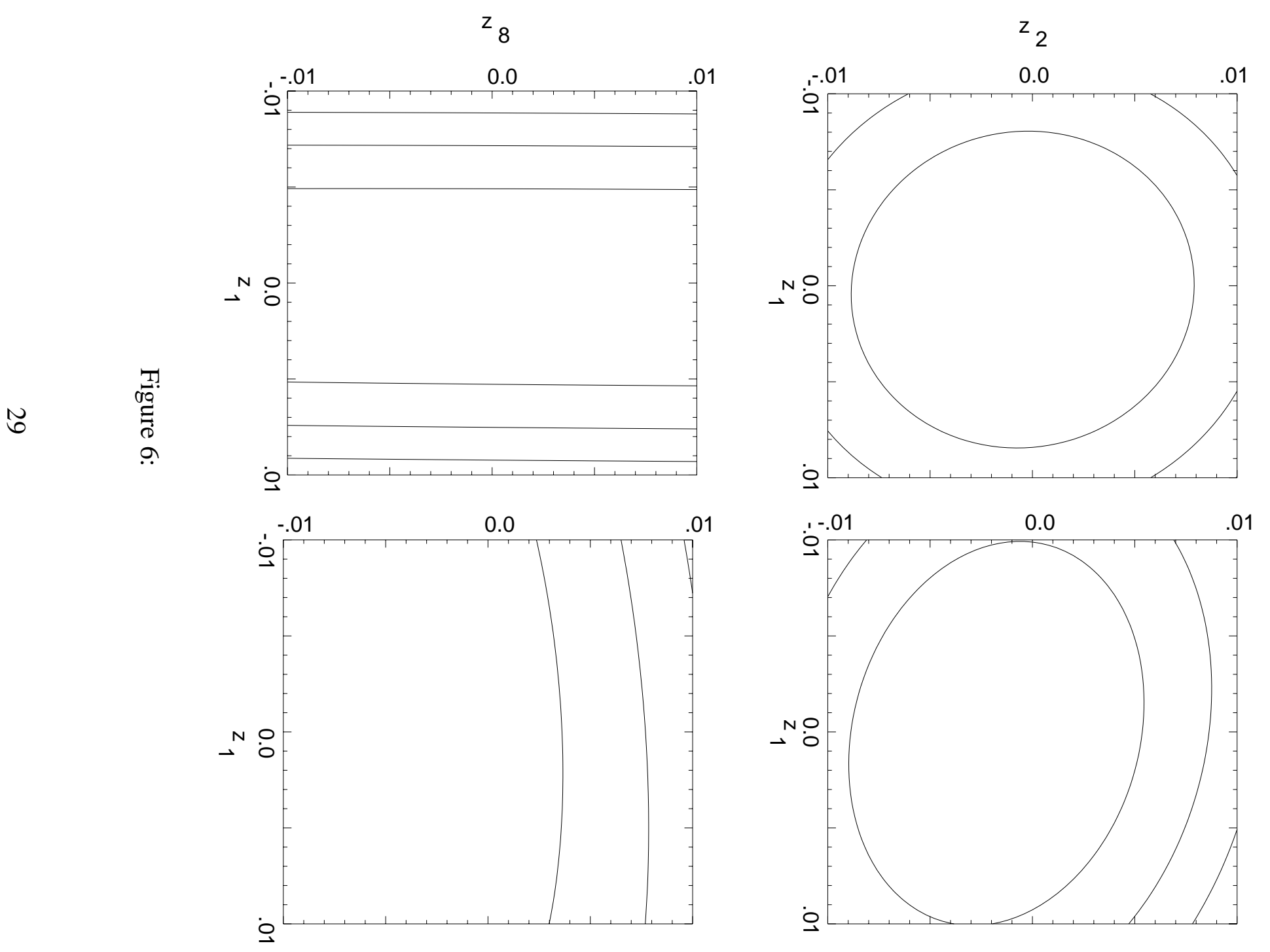

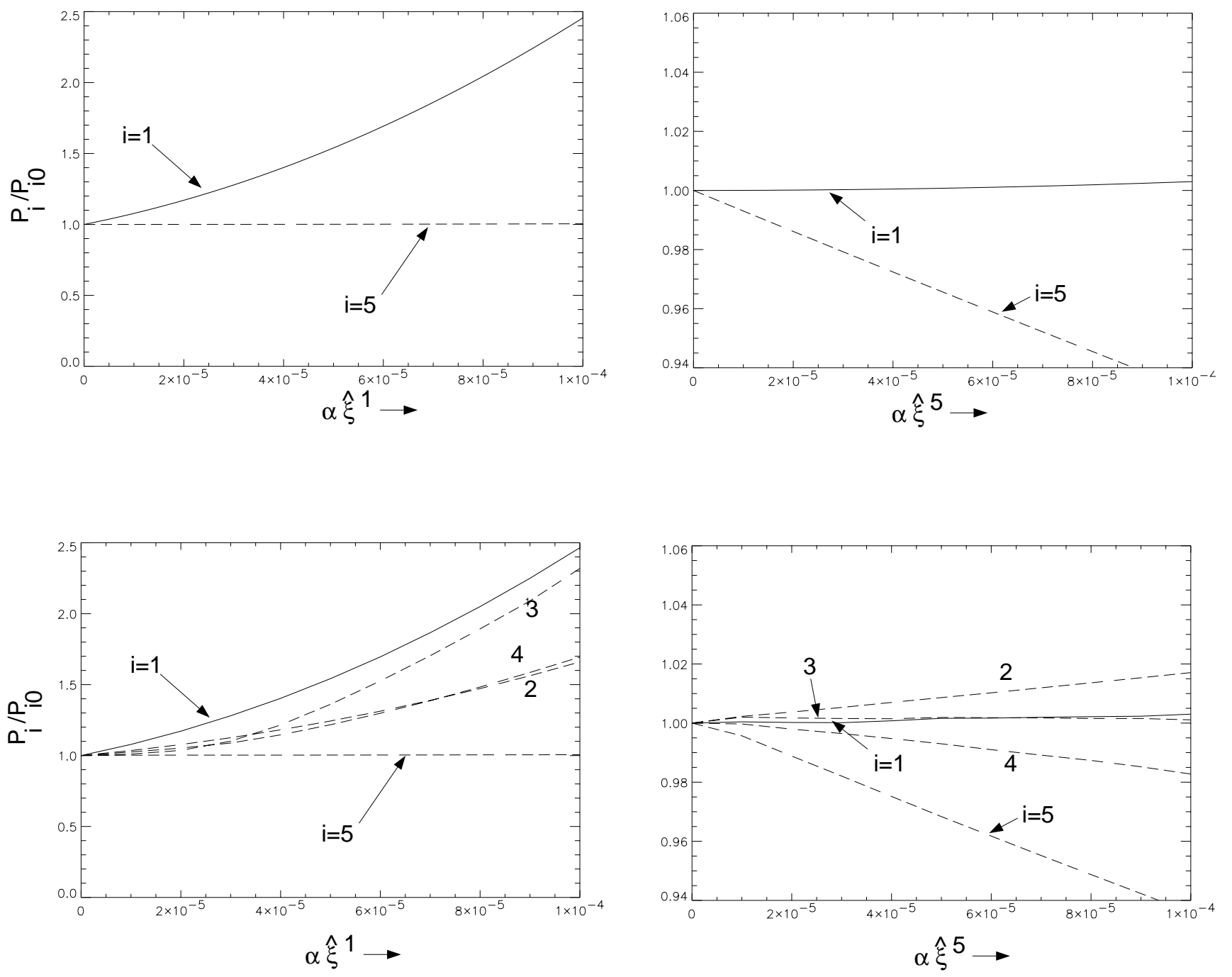

Figure 7: 

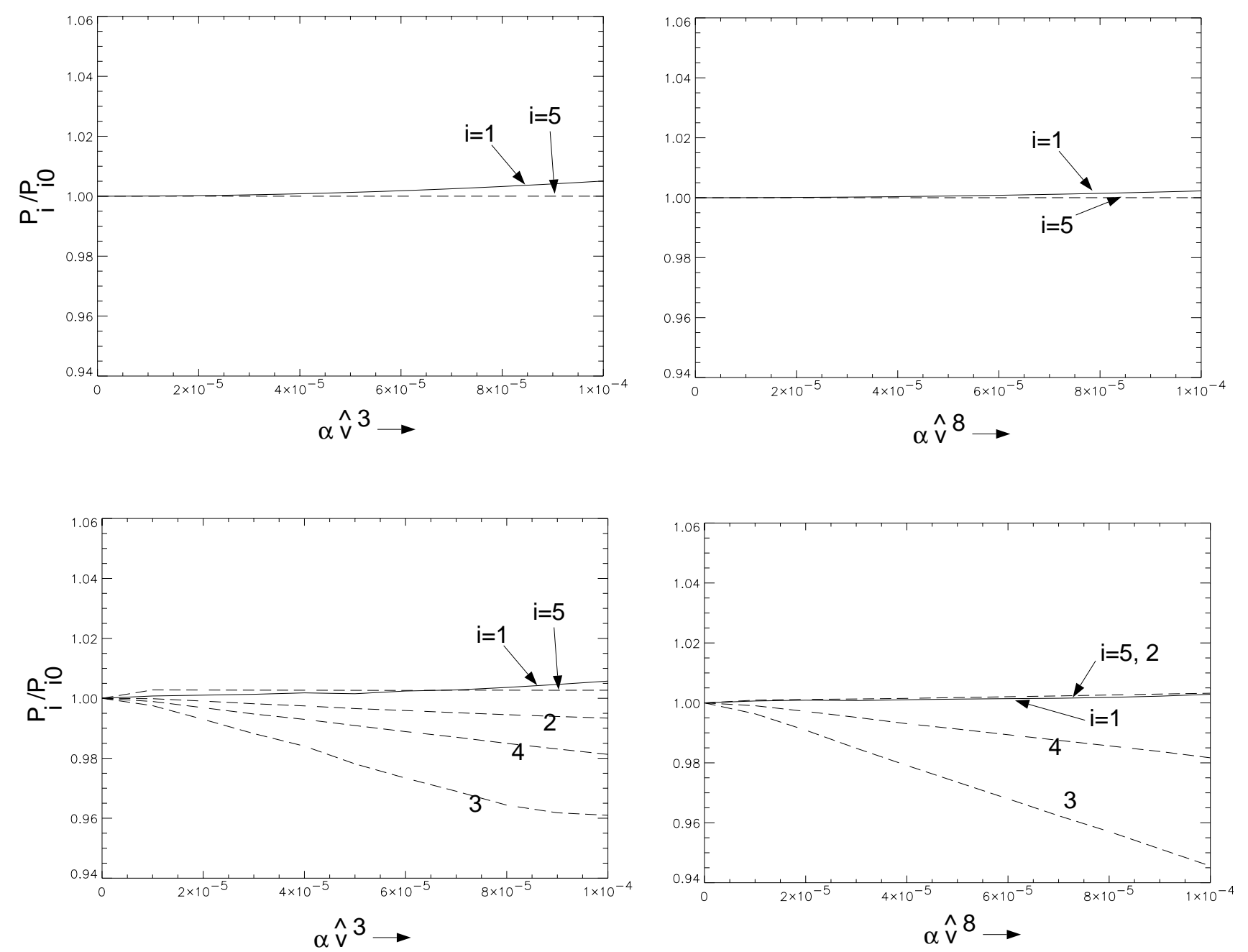

Figure 8: 

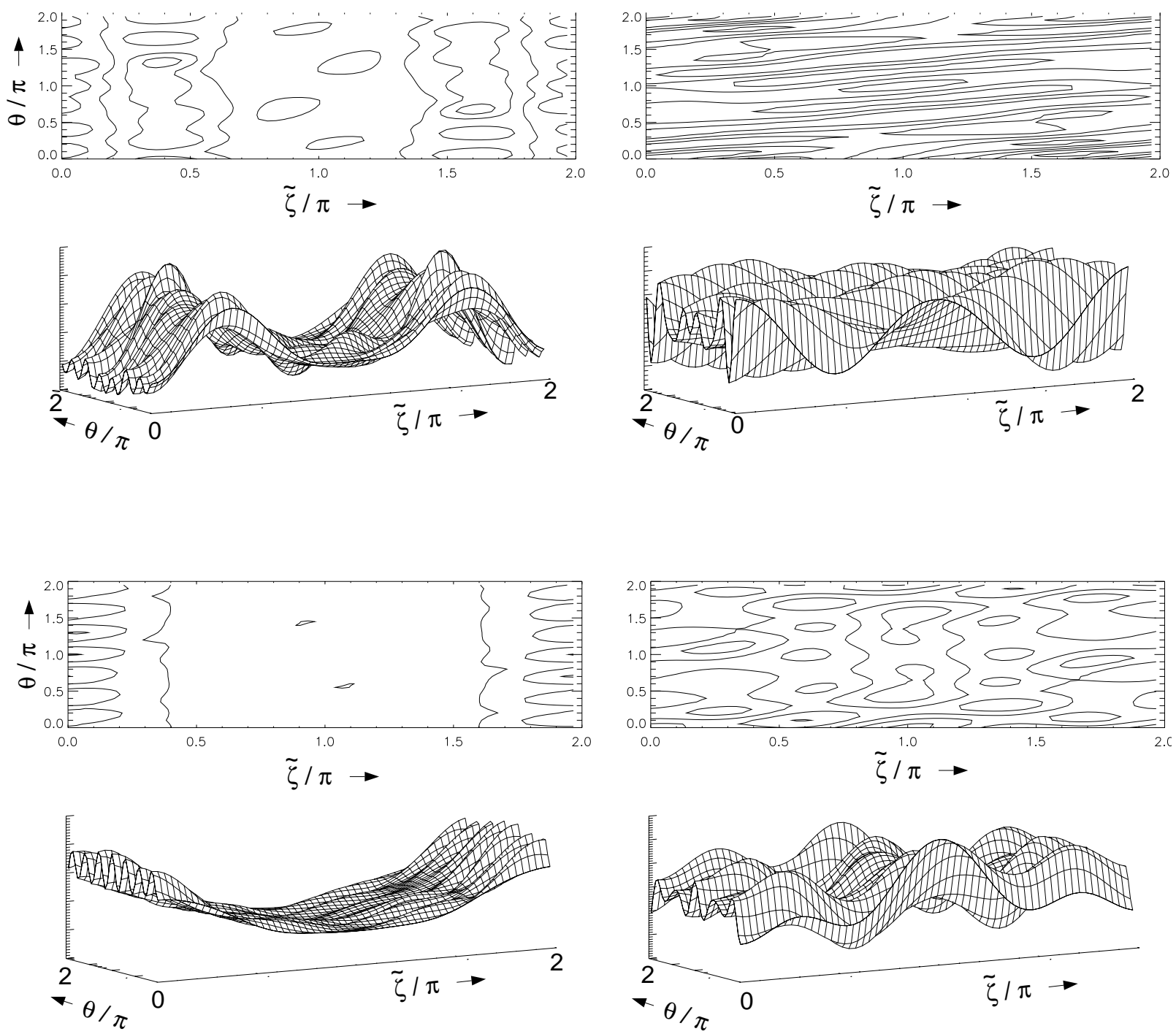

Figure 9: 

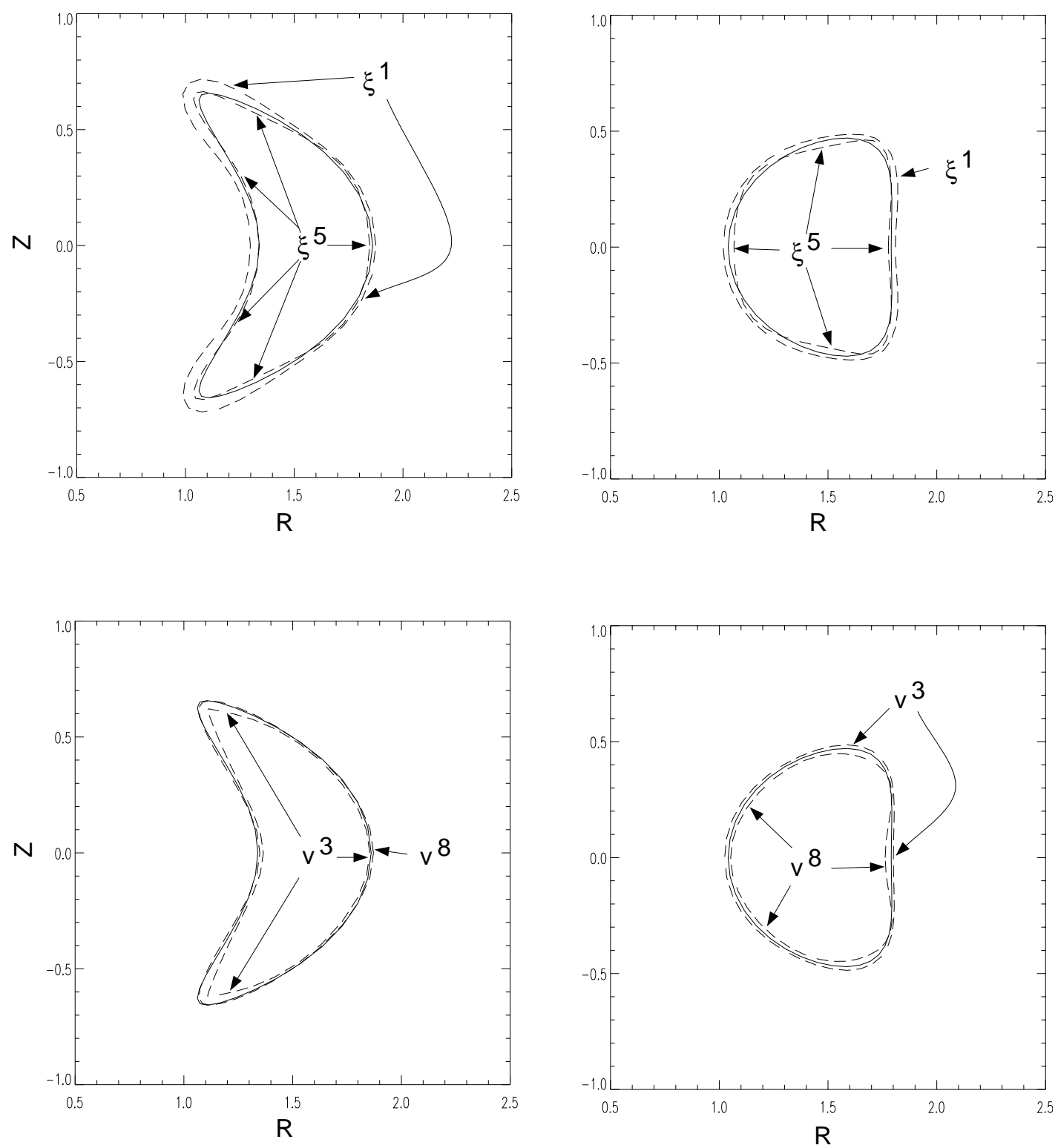

Figure 10: 


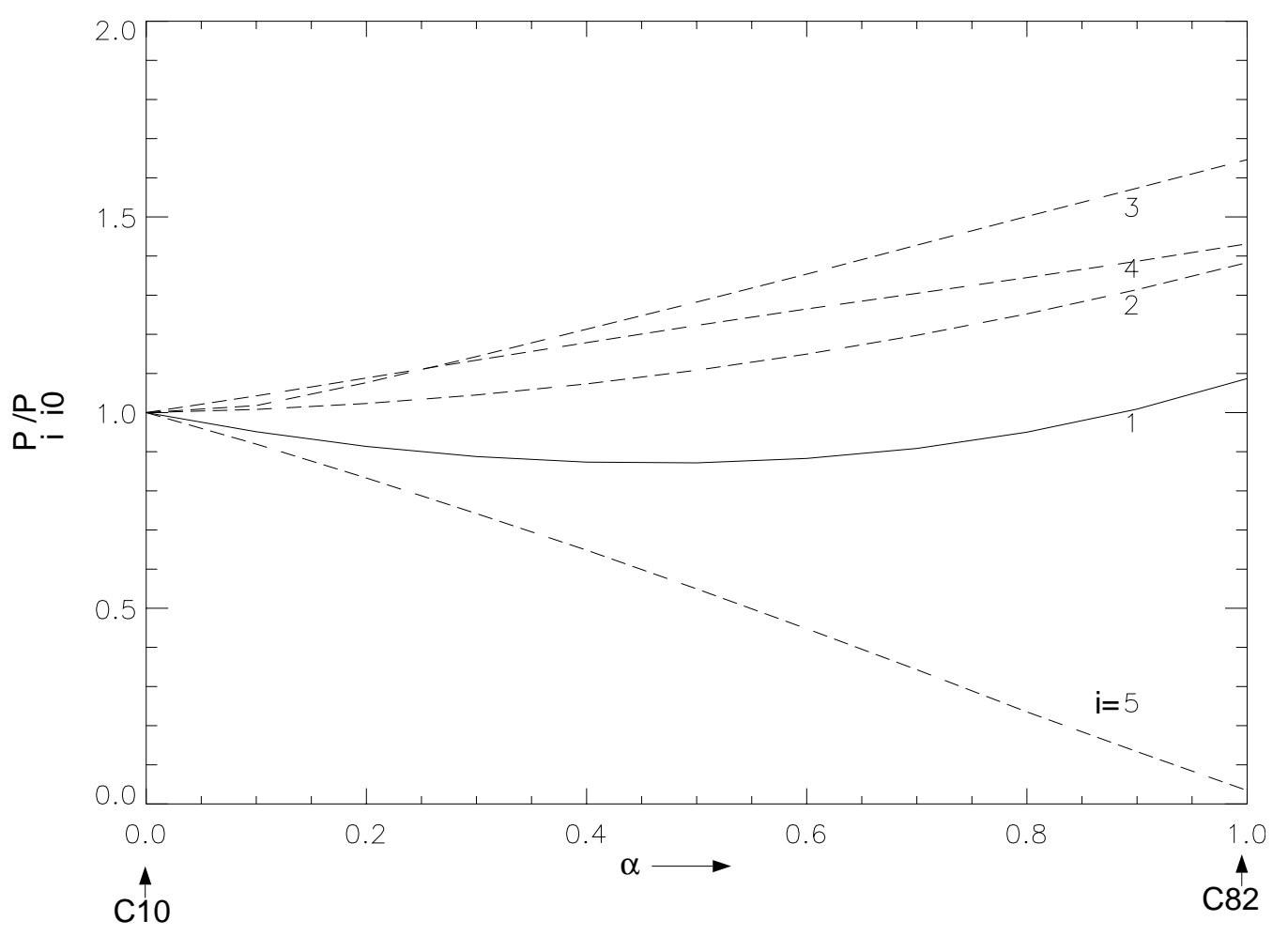

Figure 11: 

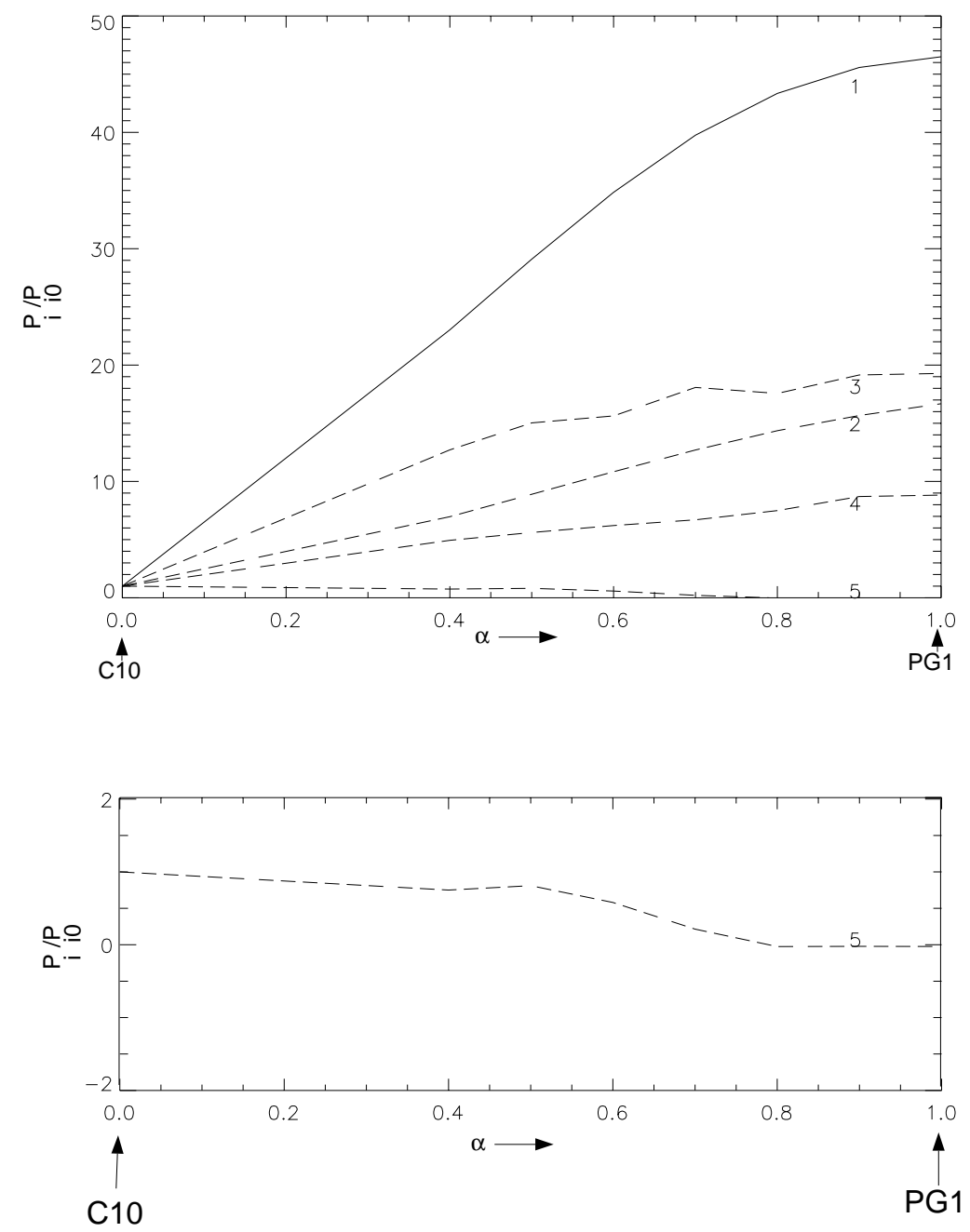

Figure 12: 